\title{
Basis of sialic acid heterogeneity in ulcerative colitis
}

\author{
J R JASS,${ }^{*} \dagger$ K SUGIHARA, ${ }^{*} \ddagger$ S B LOVE $\S$
}

From the *Department of Pathology and Imperial Cancer Research Fund Colorectal Cancer Unit, St Mark's Hospital, London, the †Department of Histopathology, Medical College of St Bartholomew's Hospital, London, the $\ddagger$ Department of Surgery, University of Tokyo, Japan, and the §Research Computing Unit, Imperial Cancer Research Fund, London

SUMMARY To test the suggestion that an inherited defect in colonic mucus rendering it susceptible to degradation by bacterial enzymes may be an important factor in the aetiology of ulcerative colitis, 650 colonoscopic and rectal biopsy specimens from 166 patients with colitis were stained by mild periodic acid Schiff (mPAS), which shows sialic acid that is deficient in O-acetyl substituents. There was an excess of mPAS positive sialic acid in patients with chronic ulcerative colitis, but the increased expression was patchy and coincided with a morphological change in the form of epithelial hyperplasia (metaplasia). Hyperplasia was more common in the rectum and in women and was associated with, and presumably secondary to, active inflammation.

It is concluded that variation in the structure of sialic acid is acquired and is therefore unlikely to be implicated in the aetiology of ulcerative colitis.

In a previous study we showed that the structure of colorectal goblet cell sialic acid varies within the general population.' Although most people secrete $\mathrm{O}$ acetyl sialic acid, around $9 \%$ express sialic acid which reacts with mild periodic acid Schiff (mPAS), a result which indicates a deficiency of side chain $\mathrm{O}$-acetyl subsituents. ${ }^{2}$ We suggested that this heterogeneity in sialic acid structure may be genetic in origin. ${ }^{\prime}$ An analogy may be drawn with fast and slow acetylator phenotypes which are distributed similarly within the population and reflect the genetically determined activities of hepatic $\mathrm{N}$-acetyl transferase.

Rhodes $e$ al proposed that ulcerative colitis may be caused by an inherited defect in colonic mucin, rendering it abnormally susceptible to degradation by bacterial enzymes. ${ }^{3}$ This would be consistent with the generally accepted view that gastrointestinal mucin has an important cytoprotective role. In this context it is known that the presence of $\mathrm{O}$-acetyl side chain substituents renders sialic acid resistant to sialidase. ${ }^{4}$ Sialic acid in colitis also shows reduced O-acetyl substitution, ${ }^{56}$ but it is unclear whether these biochemical differences are genetically determined or whether they are acquired through inflammatory change.

Although mPAS positive mucus would be expected to be more sensitive to sialidase, this has not been

Accepted for publication 6 October 1987 confirmed. The first aim of this study was therefore to determine whether mPAS reactive colorectal mucin does in fact show increased susceptibility to sialidase digestion. The second was to study the histochemical distribution of mPAS positive mucin in patients with colitis. We attempted to show whether any increased expression of mPAS positive mucus is related to inflammation, morphological changes, and duration of disease, or whether it is independent of such factors and diffusely distributed. The latter finding would support the concept that an inherited mucin defect predisposes to the development of ulcerative colitis.

\section{Material and methods}

SIALIDASE SUSCEPTIBILITY OF MPAS POSITIVE MUCIN

From a previously studied series of patients who had undergone surgery for rectal cancer ${ }^{1}$ we selected 10 patients showing mPAS negative colorectal goblet cell mucin and 10 in whom an intense reaction product was featured in the entire goblet cell population. Sections ( $5 \mu \mathrm{m}$ thick) were prepared from blocks containing normal large bowel mucosa and incubated with peanut agglutinin (PNA) (Sigma) with and without prior digestion with sialidase (Sigma type V). Following the successful removal of sialic acid by sialidase, goblet cell mucin binds PNA. ${ }^{7}$ The following technique was used:

(i) Dewax and rehydrate the tissue sections. 
(ii) Incubate at $37^{\circ} \mathrm{C}$ in $1 \mathrm{mg}$ sialidase in $50 \mathrm{ml}$ acetate buffer, $\mathrm{pH} 5 \cdot 0$, overnight. An adjacent section is incubated under the same conditions in acetate buffer but without sialidase digestion.

(iii) Wash in water.

(iv) Incubate at $37^{\circ} \mathrm{C}$ in $0.1 \% \mathrm{CaCl}$ at $\mathrm{pH} 7.8$ for 20 minutes.

(v) Wash in phosphate buffered saline for five minutes.

(vi) Block endogenous peroxidase with $3 \%$ hydrogen peroxide for five minutes.

(vii) Wash in phosphate buffered saline.

(viii) Incubate in $50 \mu \mathrm{g} / \mathrm{ml}$ horseradish peroxidase conjugated PNA for 30 minutes at room temperature.

(ix) Wash three times in phosphate buffered saline.

(x) Develop peroxidase with 3,3'-diaminobenzidine in Tris buffer, $\mathrm{pH} 7 \cdot 6$, with 4 drops of $0.3 \%$ hydrogen peroxide for 10 minutes.

(xi) Wash in tap water.

(xii) Dehydrate, clear, and mount.

Known positive and negative cases were included in each staining run as controls.

\section{DISTRIBUTION OF MPAS REACTIVITY IN COLITIC MUCOSA}

One hundred and sixty six patients with ulcerative colitis were studied ( 90 males and 76 females). Thirty four had been treated previously by total colectomy and ileorectal anastomosis. Their ages ranged from 15 to 78 years (mean 46 ). One third had had the disease for less than 10 years, one third for between 10 and 20 years, and the remainder for more than 20 years. Six hundred and fifty colonoscopic and rectal biopsy specimens were examined. This was a consecutive series from colitic patients in the St Mark's cancer surveillance programme seen between December 1984 and August 1985.

The specimens were fixed in $10 \%$ neutral buffered formalin, routinely embedded, sectioned at $5 \mu \mathrm{m}$ and two serial levels stained with haematoxylin and eosin and mild periodic acid Schiff (mPAS). ${ }^{7}$ The sections stained by haematoxylin and eosin (A group) were examined by one of the authors (JRJ) and the sections stained by mPAS (B group) by another (KS). Two features were recorded in the A group sections: the presence of hyperplastic (metaplastic) change and the presence of active inflammation (absent or present). (No dysplasia was seen). Hyperplastic changes resembled those seen in the common hyperplastic (metaplastic) polyp, but were diffuse rather than polypoid. Hyperplastic change associated with ulcerative colitis has been characterised previously: (i) crypt serration; (ii) presence of a conspicuous population of columnar cells secreting variable amounts of mucin; (iii) increased PNA binding in the supranuclear (Golgi) zone and; (iv) hyperplasia of crypt base zone (fig 1).
mPAS staining was graded for each biopsy specimen as negative, focal positive, patchy positive, weak diffuse positive, and intense diffuse positive. The results were entered into a minicomputer and subsequently transferred to a mainframe computer for analysis. The examiners were unaware of each others' findings.

\section{Results}

\section{SIALIDASE SUSCEPTIBILITY}

In both mPAS positive and negative specimens PNA binding was limited to the supranuclear (Golgi) zone of goblet cells and columnar cells. Occasionally the columnar cell cytoplasm was positive, particularly in the more immature cells of the lower crypt and crypt base. After sialidase digestion goblet cell mucin in nine of 10 mPAS positive specimens showed PNA binding; none of the $10 \mathrm{mPAS}$ negative cases was influenced by previous sialidase treatment (figs $2 \mathrm{a}-\mathrm{c}$ ).

\section{MPAS STAINING IN BIOPSY MATERIAL}

Staining with mPAS within a series of biopsy specimens from an individual patient gave a negative, diffusely positive, or discontinuously positive result. Patients in whom all goblet cells within all specimens were intensely positive with mPAS were removed from the main analyses described below. There were 11 such patients, representing a group in whom the mPAS positive reaction is probably constitutional. There

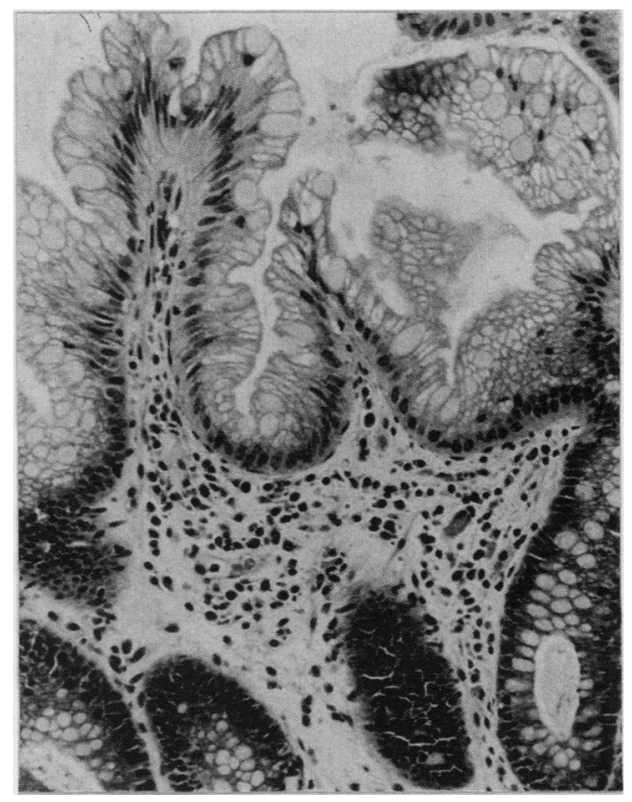

Fig 1 Hyperplastic focus in ulcerative colitis with characteristic crypt serration (Haematoxylin and eosin). 


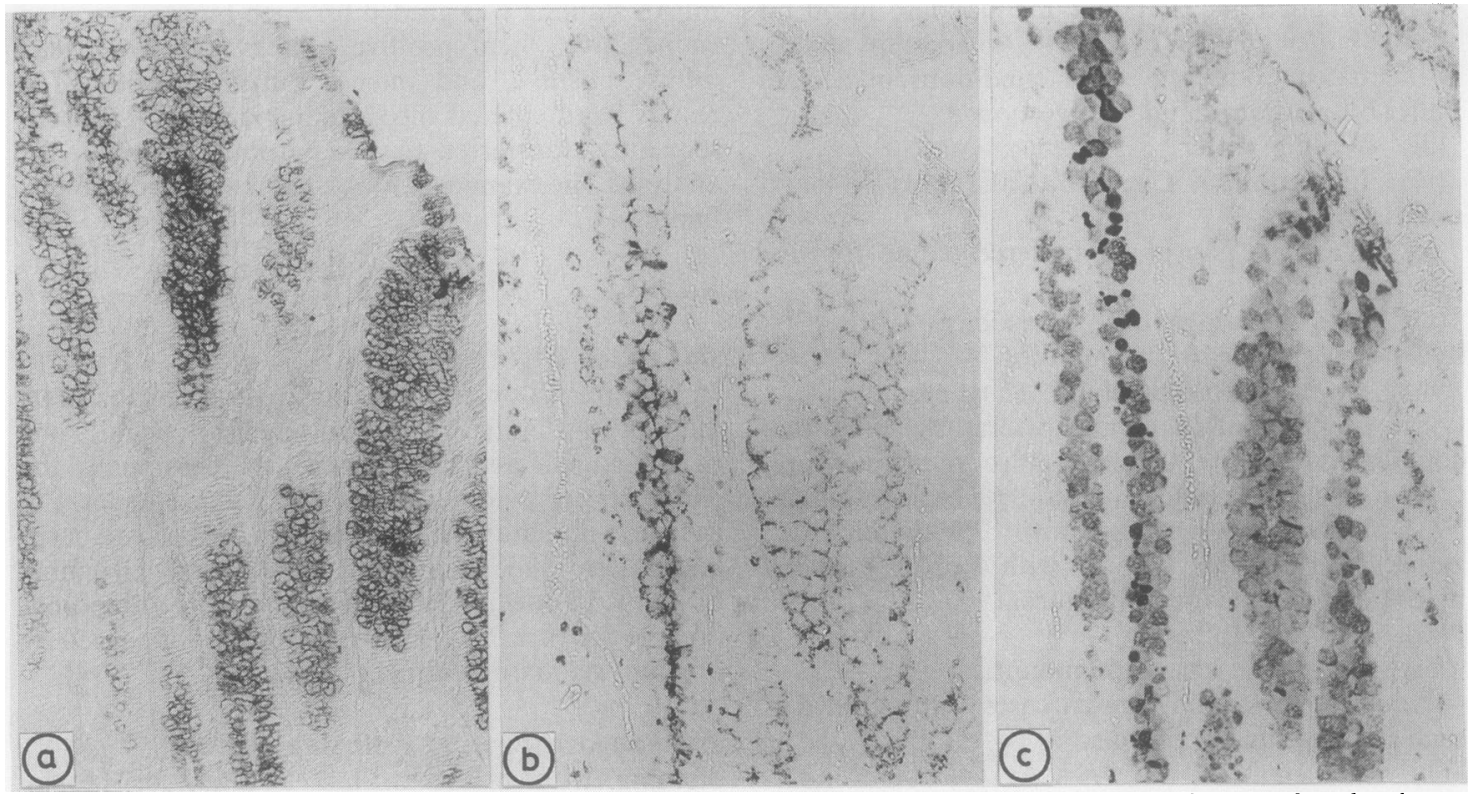

Fig 2 Section showing normal colorectal mucosa which is positive with $m P A S(a)$. Serial section has been incubated with peanut lectin which binds to sugars in supranuclear (Colgi) zone $(b)$. When incubation with peanut lectin is preceded by sialidase digestion, lectin is found to bind strongly with goblet cell mucus (c), indicating removal of sialic acid. ( (a) mild PAS; (b) peanut lectin; and (c) sialidase-peanut lectin.)

were two forms of discontinuous positivity with mPAS. In the first, groups of crypts stained intensely while others showed a weak or negative reaction within the same biopsy specimen (patchy positive

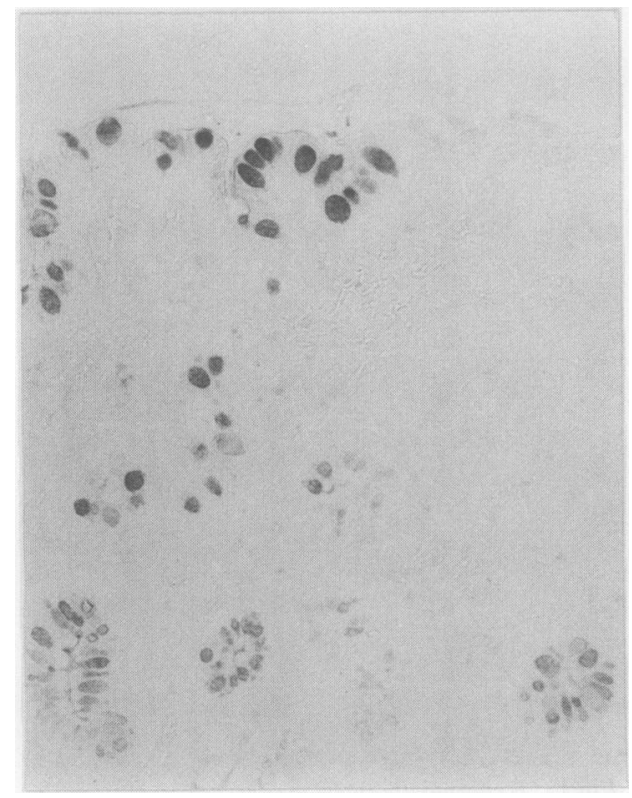

Fig 3 Patchy positive staining with $m P A S$ in colitic biopsy specimen. staining) (fig 3). In the second, some biopsy specimens from a colonoscopic series were intensely positive throughout whereas others from the same series showed patchy, weak, or no staining. The various

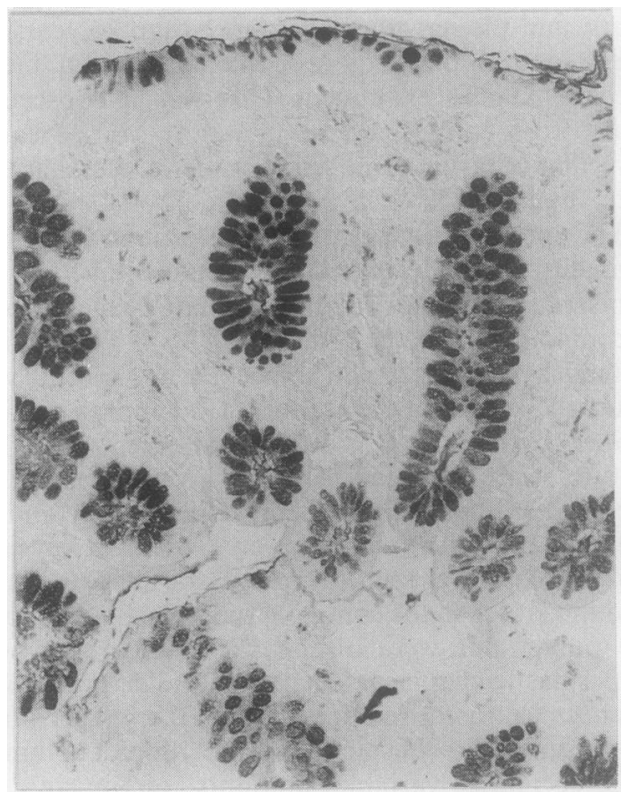

Fig 4 Intense diffuse positive staining with $m P A S$ in colitic biopsy specimen. 
Table 1 Association between $M P A S$ positivity and individual clinical and pathological variables

\begin{tabular}{|c|c|c|c|}
\hline Variable & $\chi^{2}$ test & $\begin{array}{l}\text { Degree } \\
\text { of freedom }\end{array}$ & $p$ value \\
\hline $\begin{array}{l}\text { Hyperplastic foci } \\
\text { Active inflammation } \\
\text { Site: rectum, left }\end{array}$ & $\begin{array}{r}31.8 \\
9.8\end{array}$ & $\begin{array}{l}1 \\
1\end{array}$ & $\begin{array}{l}0.001 \\
0.005\end{array}$ \\
\hline $\begin{array}{l}\text { colon, right colon } \\
\text { Duration of disease* } \\
\text { Age† } \\
\text { Sex: female > male } \\
\text { Previous colectomy and }\end{array}$ & $\begin{array}{r}12 \cdot 5 \\
16 \cdot 5 \\
14 \cdot 3 \\
5 \cdot 7\end{array}$ & $\begin{array}{l}2 \\
5 \\
4 \\
1\end{array}$ & $\begin{array}{l}0.005 \\
0.01 \\
0.01 \\
0.02\end{array}$ \\
\hline ileorectal anastomosis & 3.4 & 1 & NS \\
\hline
\end{tabular}

$* 0-5,6-10,11-15,16-20,21-25,26$ years and older

$\dagger<30,31-40,41-50,51-60,61$ years and older

staining patterns for each biopsy specimen comprised three groups: (i) negative, (ii) weak, focal, or patchy positive, and (iii) intense diffuse positive (fig 4).

Data were subjected to three forms of analysis. In the first associations between mPAS positivity and each clinical or pathological variable were assessed individually (table 1). Table 1 would indicate a dominant interaction between mPAS positivity and the presence of hyperplastic foci within the same biopsy specimen. There are smaller effects with active inflammation, site of biopsy, duration of disease, age and sex. To see if these effects were independent of hyperplastic foci analyses were undertaken separately on biopsy specimens grouped as with or without hyperplastic foci (table 2). The data indicate an important correlation between the secretion of mPAS positive mucin and hyperplasia, but little or no independent correlation with other variables. Finally, associations between hyperplasia and other clinical and pathological variables were analysed (table 3 ). For this purpose the definition of hyperplasia was refined to include not only appropriate morphological features but also the expression of mPAS positive mucin.

\section{Discussion}

Our finding that mPAS positive mucus is sensitive to sialidase is of interest in the light of the suggestion that ulcerative colitis might be caused by an inherited defect in the structure of mucus, rendering it abnormally susceptible to digestion by bacterial glycosidases. ${ }^{3}$ We succeeded in showing that the goblet cell mucus in colitic patients is changed by the increased expression of sialic acid lacking O-acetyl substituents, as shown by the mPAS technique. ${ }^{2}$ This confirms the findings of earlier reports using related histochemical techniques. ${ }^{56} \mathrm{We}$ wanted to determine, however, if this was a diffuse change and likely to be constitutional, or a patchy change relating to acquired mucosal injury. In fact, in only 11 of the 166 colitic patients $(6 \%)$ did the entire goblet cell population secrete mucin that was strongly positive with mPAS, a distribution that is similar to that of the general population.' Our group with colitis therefore shows no evidence of an excess of this inherited sialic acid variant. Many of the colitic patients have relatively mild disease, indicated by the fact that they have retained their colons. It could thus be argued that the constitutional presence of sialic acid deficient in $\mathrm{O}$ acetyl substituents might influence only the severity of the disease and would not therefore be featured in patients with mild colitis. Our series, however, included 32 patients with a previous colectomy and ileorectal anastomosis, many of whom had severe disease at a young age. In only one of these did the rectal biopsy specimen show a diffuse and intense mPAS reaction. We also studied (data not shown) colectomy material from 10 patients presenting with acute fulminating disease; again there was no excess of mPAS positivity, only one patient being implicated. Thus there is no reason to believe that constitutional sialic acid heterogeneity is related to either susceptibility to ulcerative colitis or to the severity of the disease.

We have shown that the excess mPAS positive mucin in colitic patients occurs in patches and is correlated with a histological lesion, which we have described as hyperplasia. These patches of hyperplasia are diffuse and do not present macroscopically as mucosal nodules; histologically, however, they are often indistinguishable from the common hyperplastic or metaplastic polyp. There is an interesting parallel

Table 2 Associations between MPAS positivity and variables stratified for presence or absence of hyperplastic foci

\begin{tabular}{|c|c|c|c|c|c|c|}
\hline \multirow[b]{3}{*}{ Variable } & \multicolumn{6}{|c|}{ Hyperplastic foci } \\
\hline & \multicolumn{3}{|l|}{ Present } & \multicolumn{3}{|l|}{ Absent } \\
\hline & $x^{2}$ test & Degree of freedom & p value & $\chi^{2}$ test & Degree of freedom & $p$ value \\
\hline $\begin{array}{l}\text { Inflammation } \\
\text { Site } \\
\text { Duration of disease } \\
\text { Age } \\
\text { Sex }\end{array}$ & $\begin{array}{l}1.6 \\
0.6 \\
7.6 \\
2.7 \\
1.9\end{array}$ & $\begin{array}{l}1 \\
2 \\
5 \\
2 * \\
1\end{array}$ & $\begin{array}{l}\text { NS } \\
\text { NS } \\
\text { NS } \\
\text { NS } \\
\text { NS }\end{array}$ & $\begin{array}{r}3.9 \\
6 \cdot 0 \\
8.4 \\
10 \cdot 9 \\
1 \cdot 1\end{array}$ & $\begin{array}{l}1 \\
2 \\
5 \\
4 \\
1\end{array}$ & $\begin{array}{l}0.05 \\
0.05 \\
\text { NS } \\
0.05 \\
\text { NS }\end{array}$ \\
\hline
\end{tabular}

*Age grouping: $<10,11-20$, and 21 and over as number of observations for some cells was $<5$. 
Table 3 Associations between hyperplastic foci and other clinical and pathological variables

\begin{tabular}{|c|c|c|}
\hline & No of biopsies & No with hyperplasia (\%) \\
\hline $\begin{array}{l}\text { Site: } \\
\text { Right colon } \\
\text { Left colon } \\
\text { Rectum }\end{array}$ & $\begin{array}{l}212 \\
185 \\
204 \\
\text { r linear trend }=15\end{array}$ & $\begin{array}{c}5(2.4 \%) \\
9(4.9 \%) \\
24(11 \cdot 8 \%) \\
1), p=0.001\end{array}$ \\
\hline $\begin{array}{l}\text { Active inflam } \\
\text { tion: } \\
\text { Present } \\
\text { Absent }\end{array}$ & $\begin{array}{l}133 \\
471 \\
\text { Global } \chi^{2}=15 \cdot 2(d\end{array}$ & $\begin{array}{l}18(13 \cdot 5 \%) \\
20(4 \cdot 2 \%) \\
p=0.001\end{array}$ \\
\hline $\begin{array}{l}\text { Age of patien } \\
\text { (years): } \\
<50 \\
51-60 \\
61 \text { and over }\end{array}$ & $\begin{array}{c}299 \\
144 \\
110 \\
\text { for linear trend }=8\end{array}$ & $\begin{array}{l}12(4.0 \%) \\
11(7.6 \%) \\
13(11 \cdot 8 \%) \\
1), p=0.02\end{array}$ \\
\hline $\begin{array}{l}\text { Sex of patien } \\
\text { Male } \\
\text { Female }\end{array}$ & $\begin{array}{c}331 \\
232 \\
\text { Global } \chi^{2}=15 \cdot 3(\mathrm{~d}\end{array}$ & $\begin{array}{l}10(3.0 \%) \\
26(11.2 \%) \\
p=0.001\end{array}$ \\
\hline
\end{tabular}

The total number of biopsies varies slightly as a result of information being unobtainable.

with epithelial dysplasia which usually presents as a diffuse lesion in colitic patients and as a circumscribed lesion (adenoma) in patients without colitis. Like the hyperplastic polyp, diffuse hyperplasia in colitic patients is most common in the rectum followed by the left and right colon (table 3). Hyperplasia was diagnosed in $12 \%$ of the randomly sampled rectal biopsy specimens and is clearly a common occurrence in patients with chronic colitis. The change is probably a reversible sequela to mucosal injury. Indeed, hyperplasia is associated with the presence of active inflammation (table 3), though is also observed in its absence. Unlike the hyperplastic polyp, colitic hyperplasia seems to be more prevalent in women (table 3 ).

Although the precise histopathogenesis of epithelial hyperplasia is not understood, it is clear that the lesion must be acquired. As the mucin changes observed in colitic patients coincide with the presence of hyperplasia and show either no further or only weak interdependence with other variables (table 2) it is logical to infer that the change in sialic acid structure is also acquired. It is unlikely that the acquired modifications in sialic acid structure associated with epithelial hyperplasia would affect the course of the disease. The findings of this study emphasise the importance of studying functional changes not in isolation but in association with morphology.

The histological material and clinical data used in this study were provided by Professor J Lennard-Jones, Dr J Ritchie, and Dr C B Williams.

\section{References}

1 Sugihara K, Jass JR. Colorectal goblet cell sialomucin heterogeneity and its relation to malignant disease. J Clin Pathol 1986;39:1088-95.

2 Veh RW, Meessen D, Kuntz D, May B. Histochemical demonstration of side-chain substituted sialic acids. In: Malt RA, Williamson RCN, eds. Colonic carcinogenesis. Lancaster: MTP Press Ltd, 1982:355-65.

3 Rhodes JM, Black RR, Gallimore R, Savage A. Histochemical demonstration of desialation and desulphation of normal and inflammatory bowel disease rectal mucus by faecal extracts. Gut 1985;25:1312-8.

4 Culling CFA, Reid PE, Clay MG, Dunn WL. The histochemical demonstration of $\mathrm{O}$-acylated sialic acid in gastrointestinal mucins: their association with the potassium hydroxideperiodic acid Schiff effect. J Histochem Cytochem 1974;22: 826-31.

5 Reid PE, Culling CFA, Dunn WL, Ramey CW, Clay MG. Clinical and histochemical studies of normal and diseased human gastrointestinal tract. I. A comparison between histologically normal colon, colonic tumors, ulcerative colitis and diverticular disease. Histochem J 1984:16:235-51.

6 Culling CFA, Reid PE, Dunn WL. A histochemical comparison of the O-acylated sialic acid of the epithelial mucins in ulcerative colitis, Crohn's disease and normal subjects. J Clin Pathol 1979;32:1272-7.

7 Cooper HS. Peanut lectin binding sites in large bowel carcinoma. Lab Invest 1982;47:383-90.

8 Jass JR, England J, Miller K. Value of mucin histochemistry in follow up surveillance of patients with long standing ulcerative colitis. J Clin Pathol 1986;39:393-8.

Requests for reprints to: Dr J R Jass, St Mark's Hospital, City Road, London ECIV 2PS, England. 